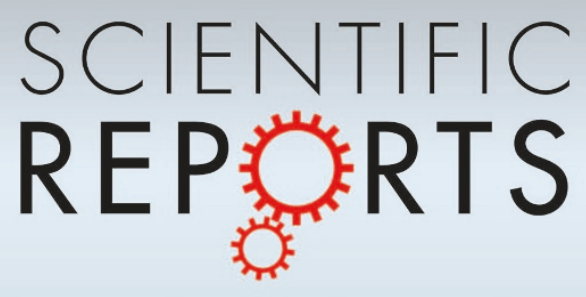

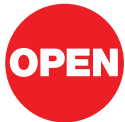

SUBJECT AREAS: TISSUE ENGINEERING

TISSUES

CARDIOVASCULAR MODELS

REGENERATIVE MEDICINE

Received

1 October 2012

Accepted

25 January 2013

Published

19 February 2013

Correspondence and requests for materials should be addressed to T.O. (tokano@abmes. twmu.ac.jp)

* These authors contributed equally to this work.

\section{In Vitro Engineering of Vascularized Tissue Surrogates}

\author{
Katsuhisa Sakaguchi i *, Tatsuya Shimizu2*, Shigeto Horaguchi' , Hidekazu Sekine ${ }^{2}$, Masayuki Yamato², \\ Mitsuo Umezu' \& Teruo Okano²
}

\begin{abstract}
'School of Creative Science and Engineering, TWIns, Waseda University, 2-2 Wakamatsu-cho, Shinjuku-ku, Tokyo 162-8480, Japan, ${ }^{2}$ Institute of Advanced Biomedical Engineering and Science, TWlns, Tokyo Women's Medical University, 8-1 Kawada-cho, Shinjuku-ku, Tokyo 162-8666, Japan.
\end{abstract}

In vitro scaling up of bioengineered tissues is known to be limited by diffusion issues, specifically a lack of vasculature. Here, we report a new strategy for preserving cell viability in three-dimensional tissues using cell sheet technology and a perfusion bioreactor having collagen-based microchannels. When triple-layer cardiac cell sheets are incubated within this bioreactor, endothelial cells in the cell sheets migrate to vascularize in the collagen gel, and finally connect with the microchannels. Medium readily flows into the cell sheets through the microchannels and the newly developed capillaries, while the cardiac construct shows simultaneous beating. When additional triple-layer cell sheets are repeatedly layered, new multi-layer construct spontaneously integrates and the resulting construct becomes a vascularized thick tissue. These results confirmed our method to fabricate in vitro vascularized tissue surrogates that overcomes engineered-tissue thickness limitations. The surrogates promise new therapies for damaged organs as well as new in vitro tissue models.

 issue engineering methods have promised new cell-based therapies for defective organs through regeneration, and new tissue-analogue models for drug testing and biomedical and physiological research. However, facile procedures for preparing engineered 3-D tissues are made difficult by a lack of intrinsic vascularity and transport systems to nourish such engineered tissues: in vitro viable tissue-like systems often exhibit dimensions beyond practical perfusion limits, and have no functional blood vessels with flowing blood to supply nutrients and oxygen, and to remove waste products. Most approaches to engineer thick tissue are performed in vivo using the body of small animals as a living incubator to produce natural vascular networks ${ }^{1-3}$. This in-body reactor approach has many practical limitations for most envisioned applications.

Our laboratory has fabricated living cardiac cell sheets in $v i v 0^{4,5}$. This entire process of fabricating living, dense cellular constructs, called "Cell Sheet Engineering", has been demonstrated with several cell types ${ }^{6-9}$. Such layered cell sheets stratify tightly because of the preserved native extracellular matrix present on individual cell sheets, facilitating rapid establishment and maintenance of cell-cell communication among the layered cell sheets ${ }^{10,11}$. This cell sheet engineering technology has the potential to fabricate unique, functional cell-dense cell sheets for treating damaged and non-functional organ $\mathrm{s}^{12}$ if problems including hypoxia, nutrient insufficiency, and waste accumulation ${ }^{13,14}$ are overcome. Therefore, cardiac cell sheets were transplanted into the subcutaneous tissue of nude rats, and these transplanted cell sheets were found to be preserved in vivo for extended time frames ${ }^{5}$. Key to such preservation and viability in vivo appears to be the adequate vascularization that allows host blood to perfuse the newly produced microvessels within the cell sheet. Rapid host perfusion supplies sufficient amounts of oxygen and nutrients to the implanted tissues. Therefore, to establish 3-D tissue engineering, nutrient and oxygen supplies which be perhaps supplied by biologically scavenging the host's vascular system, is essential. However, there is, as yet, no adequate bioreactor system to provide the necessary perfusion via vascularization in multi-layered cell sheets.

\section{Results}

This study describes a newly designed approach to a bioreactor for engineering vascularized cardiac tissue based on the concept that layered cell sheets can culture successfully and reliably on an artificial culture having microchannels simulating in vivo subcutaneous transport and perfusion conditions. To realize this idea, a new perfusion bioreactor system for culturing 3-D cell-dense cardiac cell sheets on collagen-gel fabricated with imbedded microchannels able to mimic a subcutaneous vascular structure (Fig. 1a). After a triple-layered cultured 
a

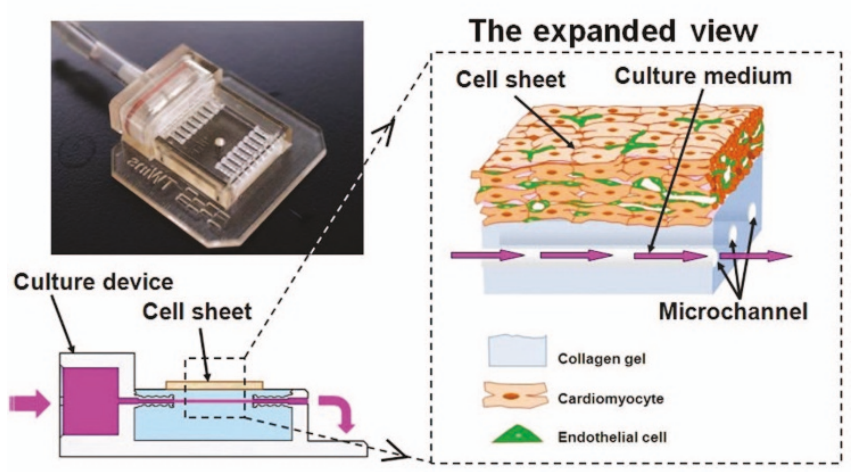

b

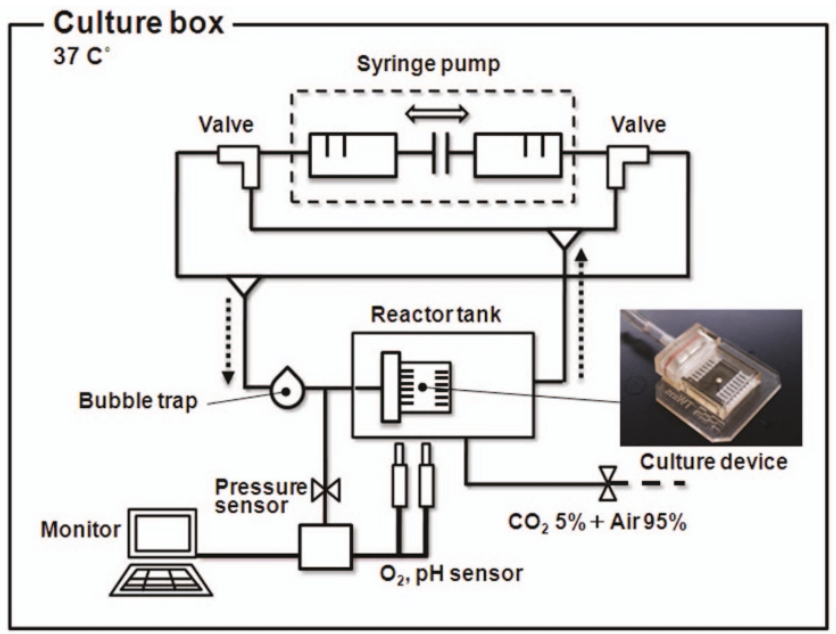

Figure $1 \mid$ Culture device and perfusion culture system. (a) The collagen-gel base with microchannels imitates the conditions of a subcutaneous structure. In the right-hand expanded view, the blue area shows collagen-gel that can imitate a subcutaneous extracellular matrix, the pink arrows indicate the direction of culture medium flow imitate blood and tissue fluid. The culture medium can diffuse into the collagen gel and provide oxygen and nutrients to the cell sheet. The left picture shows a photograph of the culture device. (b) The circuit of culture system allows the culture medium to flow in the microchannels and monitors the $\mathrm{pH}$ and oxygen conditions of the culture medium as well as using partial pressure sensors. The culture medium was perfused into the culture device at $0.5 \mathrm{~mL} / \mathrm{min}$ by a syringe pump. The oxygen and $\mathrm{pH}$ were monitored with an optic sensor to verify the conditions of the culture medium.

cardiac cell sheet, that included endothelial cells, was placed over the collagen-base with microchannels, perfusion through the collagen-based microchannels was investigated, and effects of cytokines on vascularization were also studied. During incubation, a micro-syringe pump provided a constant flow of $0.5 \mathrm{~mL} / \mathrm{min}$ to the bioreactor (Fig. 1b). After specific intervals of time under bioreactor perfusion, the cell sheet was removed and subjected to histological analysis. Specimens of these perfused cell sheets were stained by AZAN and compared to non-perfused cell sheet controls. AZAN sections of control cell sheets cultured without perfusion of culture medium demonstrated many faded color areas, indicating overall necrosis (Fig. 2a). By contrast, cell sheets with perfusion of medium showed both normal and healthy cell morphology within the cell sheets (Fig. 2b). In addition, triplelayered cell sheets were cultured with vascular endothelial growth factor (VEGF) in the culture medium. In that case, some migrating cells were observed (Fig. 2c). To better stimulate growth of vascular structures, cell sheets were also cultured in media that combined basic fibroblast growth factor (bFGF) and VEGF. Large numbers of cells migrated to create tubular structures with lumens within the collagen-gel base (Fig. 2d). Migrating cell areas in perfusion culture systems were significantly larger than that in static culture system. The areas in perfusion culture containing both VEGF and bFGF were significantly larger than those in perfusion culture systems without two growth factors and only with VEGF (Fig. 2e). To evaluate tissue viability, Live/Dead assay was performed in the static culture and in the perfusion culture with both VEGF and bFGF. Most of the cells died in the static condition in consistent with the AZAN staining image, whereas most of cells survived in the perfusion culture (Fig. 2f).

Next, green fluorescent protein (GFP)-expressing endothelial cells were replaced in culture-produced cardiac cell sheets to assist in identifying the appearance of endothelial cells. Co-cultured GFPpositive endothelial cells formed throughout the micro capillary network, as seen in the top views (Fig. $3 \mathrm{a}-\mathrm{c}$ ). In the cross-sectional views, GFP-positive endothelial cells cultured within the cardiac cell sheets were directly observed at luminal surfaces of newly formed microvessels in the constructs; similar to the luminal surfaces of microvessel vascular networks in vivo (Fig. $3 \mathrm{~d}, \mathrm{e}$ ).

To verify the capillary-like fluid-carrying functions of the collagen microchannels, fresh rat red blood cells were perfused into the bioreactor immediately prior to the removal and fixation of the cell sheets from culture. In the bioreactor controls lacking endothelial cells, the microchannels are clearly observed as flow lines stained from the red blood cells (Fig. 4a). On the other hand cultured cell sheets containing endothelial cells show rat blood cells distributed across the cell sheets similar to actual subcutaneous vessels (Fig. 4b). After red blood perfusion, cultured cardiac cell sheets, with and without endothelial cells, were removed and analyzed histologically. Neither vascular structure nor red blood cell distribution was observed in the construct without endothelial cells (Fig. 4c). By contrast, red blood cells locate consistently to the lumens of vascular networks in the construct with endothelial cells, indicating that newly created vascular networks must spontaneously anastomose with the bioreactor's collagen-based microchannels to achieve connectivity (Fig. 4d-f). Moreover, in order to capture the shape of the newly created vascular networks, a cast was taken by perfusing an epoxy resin into the bioreactor. The cast of the vascular network showed that newly created vascular networks anastomosed with the bioreactor's collagen-based microchannels (Fig. 4g, h). This significantly demonstrates that: (1) the proposed and presumed newly created vascular networks observed within cultured cell sheets are indeed capable of reliable erythrocyte transport, just as capillaries can in vivo; (2) these cell sheet-based vascular networks effectively anastomose with the collagen-based microchannels in the bioreactor and exchange their fluid contents; and (3) fabrication of viable cardiac cell sheets with perfusable, functional vascular networks was successful. Significantly, this new capability will facilitate the production of thicker tissues.

Overcoming the intrinsic mass transport- and thickness-based limitations required for multi-layered cardiac cell sheet applications has been our focus, extending this vascular network proof-of-concept within the perfusion bioreactor to thicker sheets. Neonatal rat cardiomyocyte cell sheet constructs with varying numbers of cell layers (i.e., 1 to 6) were prepared and cultured individually. After 5-day culture in the perfusion bioreactor, each cell sheet construct was removed and fixed, and the thickness of each harvested construct was measured by microscopy (Fig. 5a-f). The total thickness of cell sheet constructs increases linearly up to 3 cell sheet layers, reaching a plateau for further cell sheet layering in vitro (i.e., 4-6 layers, see Fig. 5j). This was important to show the thickness limitations that hinder the fabrication of in vitro cell sheet-based tissue by layered cardiomyocyte sheets using a single-step sheet stacking and culture process. 

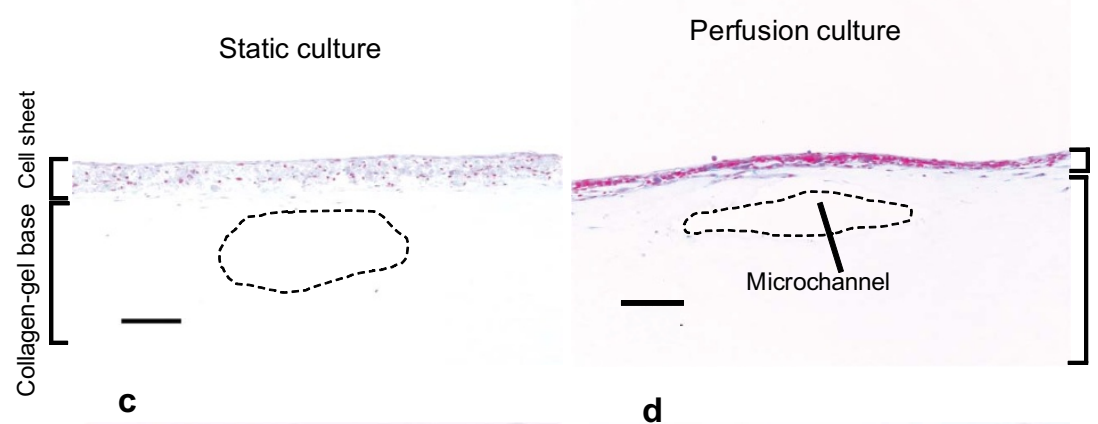

d

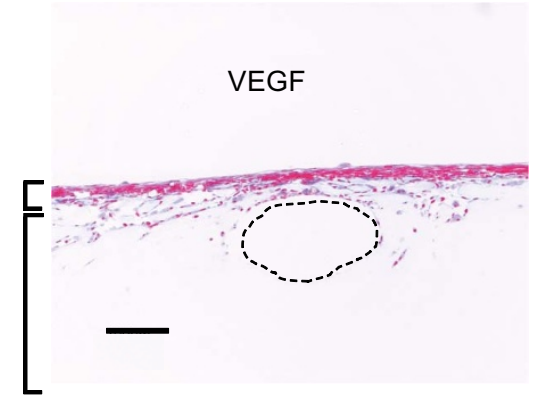

e
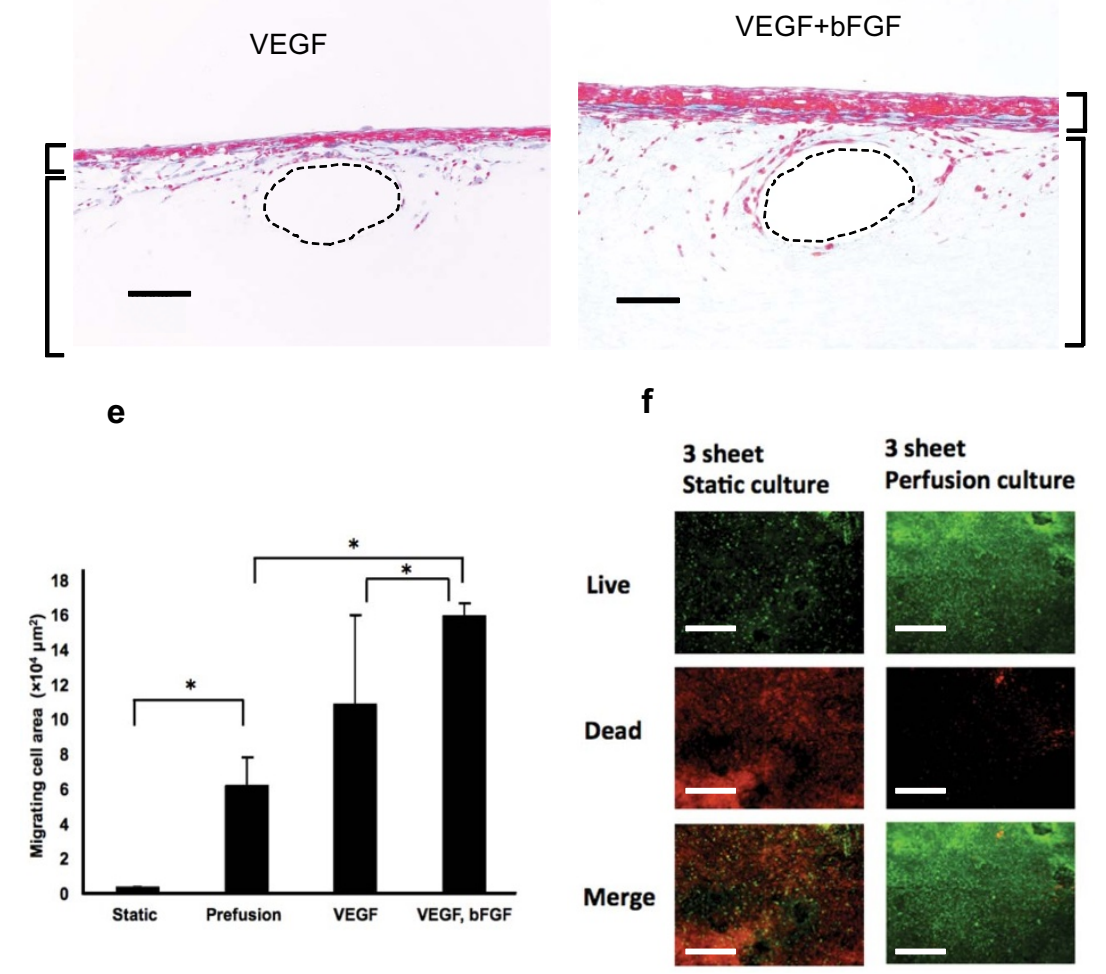

Figure $2 \mid$ Cell migration from the cell sheet and tissue viability. The specimens were stained with AZAN. In the photographs (a) (d), the upper layers are triple-layered cell sheets, and the lower part is collagen gel. The dotted-line circles are the microchannels. (a) Histological observation shows a triple-layered cell sheet on the collagen-gel cultured for 5 days without perfusion. After 5 days of static cultivation, the cell sheet had necrosed throughout. (b) A triple-layered cell sheet was cultured with perfusion at $0.5 \mathrm{~mL} / \mathrm{min}$ for 5 days. The cell sheet construct was able to survive and several cells migrated into the collagen gel. (c) With the addition of vascular endothelial growth factor (VEGF), AZAN section showed that many cells had migrated and can be seen between the cell sheets and the microchannels. (d) VEGF and basic fibroblast growth factor (bFGF) stimulated triple-layered cell sheets on the collagen-gel. A large number of cells were found to migrate and create lumens in the collagen-gel base. (Scale bar, $100 \mu \mathrm{m})(\mathrm{e}) \mathrm{Migrating}$ cell areas were counted under the conditions in (a) (d). The areas in perfusion culture systems were significantly larger than that in static culture. The areas in perfusion culture containing both VEGF and bFGF were significantly larger than those in perfusion cultures without two growth factors and only with VEGF. $\left({ }^{*} P<0.05, \mathrm{n}=3\right)$ (f) Live/Dead assay was performed in the static culture and perfusion culture for the 3-layer cardiac cell sheet. Fluorescence micrograph shows cells stained by $5 \mu \mathrm{mol} / \mathrm{L}$ calcein-AM (green $=$ live; top images) and $0.5 \mu$ mol/L ethidium homodimer- 1 (red $=$ dead; middle images). Bottom images are a merged version of the Live/Dead images. Most of the cells died in the static condition, consistent with the AZAN staining image. On the other hand, most of cells survived in the perfusion culture (Scale bar, $20 \mu \mathrm{m}$ ).

To overcome this fabrication thickness limitation for layered cardiac cell sheets, a delayed interval layering culture process was performed with 3-layer cell sheets. First, a triple-layer cell sheet was cultured in the collagen-gel microchannel bioreactor for 5 days. The resulting sheet thickness was $24 \pm 4 \mu \mathrm{m}(\mathrm{n}=3)$. A second triple-layered cell sheet was then placed directly over the first 3-layer cell sheet in the bioreactor, yielding a 6-layer cell sheet, and this thicker cell sheet construct was further incubated in the bioreactor for 5 days (Fig. 5g). The 6-layer cell sheet was synchronously beating on the collagen-gel base (Supplementary Movie). Subsequently, a third 3-layer cell sheet was placed over the 6-layer cell sheet in the bioreactor, producing a 9-layer cell sheet. This thicker cell sheet construct was incubated again for another 5 days (Fig. 5h). This process was repeated again, yielding a 12-layer cell sheet, and incubated again for 5 days (Fig. 5i). This multilayer-stacking-culture experiment was repeated three additional times. During each layer stacking process, the average thicknesses of the 3-, 6-, 9-, and 12layer cell sheet construct increased to $24 \pm 4,35 \pm 2,65 \pm 8$, and 

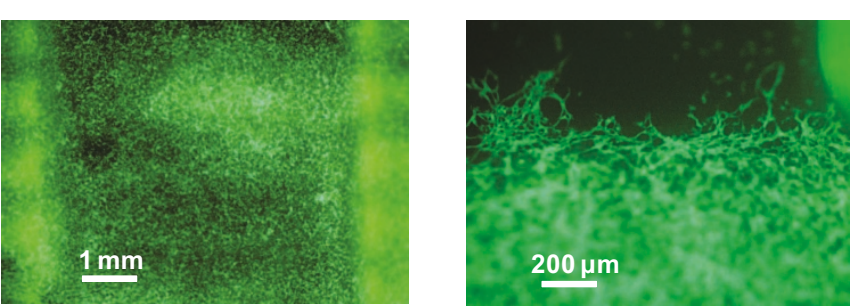

C
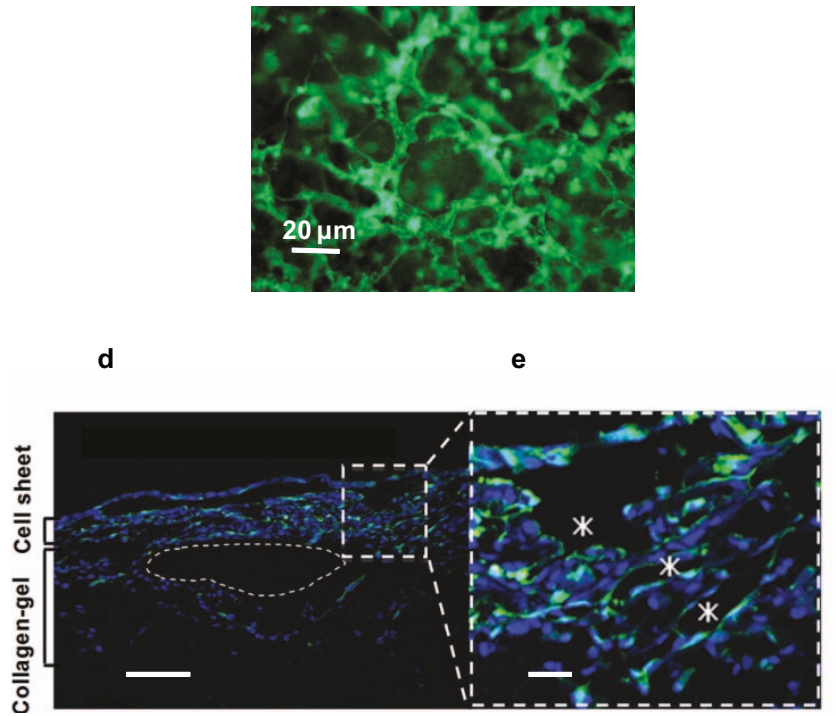

Figure 3 Geometry of endothelial cells of the cell sheet. (a) (c) GFPexpressing endothelial cells were isolated from GPF neonatal rat heart and replaced with normal endothelial cells of the cardiac cell sheet by a magnetic cell sorter technique. These images are the top views of a 3-layer cardiac cell sheet after 5 days in perfusion culture under a microscope. GFP-expressing endothelial cells are seen as green. (a) Low magnification of the center of the construct. (b) Meddle magnification of edge in the construct. (c) High magnification of the center of the construct. (d) The specimen shows that GFP-positive cells exist at the lumen surface. Blue signals indicated nuclei. (Scale bar, $100 \mu \mathrm{m}$ ). (e) The right photograph shows the expanded view of the square area in (d) (Scale bar, $20 \mu \mathrm{m}$ ).

$110 \pm 4 \mu \mathrm{m}(\mathrm{n}=3)$, respectively (Fig. 5k). Histological analysis of anti-troponin $\mathrm{T}$ antibody staining revealed stratified cardiomyocytes in the 6-layer construct (Fig. 5l). HE stained sections of the multilayer cell sheet constructs showed that the 12-layer cell sheet was well integrated without any observable necrotic tissue. A Live/Dead assay showed that most of the cells survived, but that significant cell death was focally observed within the 12-layer tissue (Fig. 5m). This multistep layering procedure also provided vascularization within the cardiac tissue-like layers which exceeded $110 \mu \mathrm{m}$ in thickness, well-beyond the diffusive transport limits for cells. During the initial 5-day incubation period for the first triple-layered cell sheet, endothelial cells from the cell sheet were observed to migrate into the collagen gel channels, forming vessels that eventually spontaneously anastomosed to the bioreactor microchannels enabling the requisite supply of media containing both oxygen and cell nutrients. Importantly, during further 5-day incubations after each new layer in the serial cell sheet stacking process (i.e., forming cell sheet constructs 6- to 12-layers thick), blood vessel precursor structures were directly observed throughout the constructs extending from the initial triple-layered cell sheet adjacent to the microchannels.

These key results for stimulating endogenous vascularization in an artificial cell culture system are unique, and provide critical new a

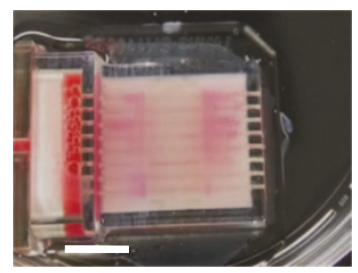

c

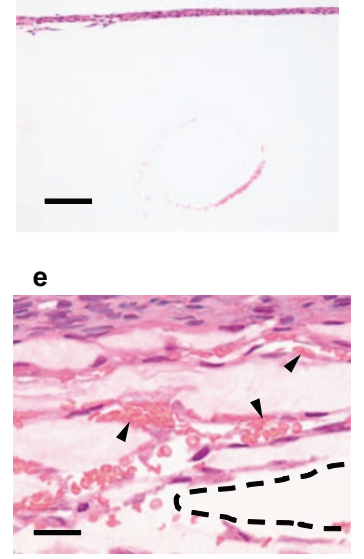

g

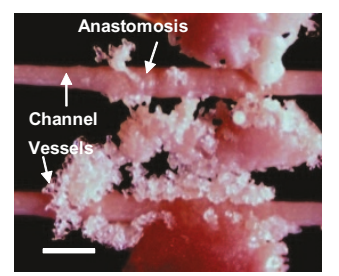

b

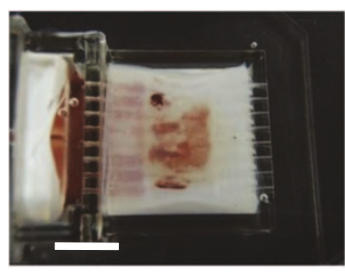

d

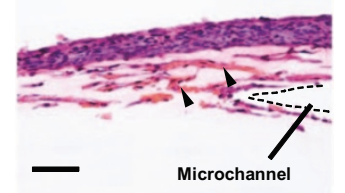

f

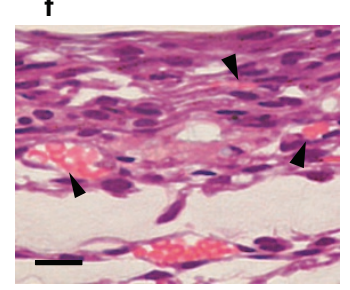

h

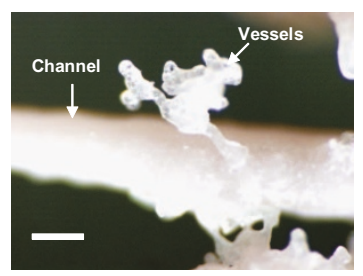

Figure $4 \mid$ Red blood cells and resin perfusion into the endothelial lumen. (a) When the device has no endothelial cells, the flow line of red blood cells is clearly observed to follow on the microchannels (Scale bar $1 \mathrm{~cm}$ ). (b) When endothelial cells are included, rat blood cells spread throughout the cell sheets like real subcutaneous vessels (Scale bar, $1 \mathrm{~cm}$ ). (c) HE stained section shows no migrating cells in the construct without endothelial cells (Scale bar, $200 \mu \mathrm{m}$ ). (d) HE stained section of the construct containing endothelial cells shows a lot of migrating cells and a vascular formation. The red blood cells flowed into the newly created vascular network (Scale bar, $200 \mu \mathrm{m}$ ). (e) In the high magnification image, arrowheads indicate that red blood cells locate consistently to the lumens of the vascular networks between the microchannel and the cell sheet. The dotted line indicates the collagen-based microchannel (Scale bar, $50 \mu \mathrm{m}$ ). (f)Red blood cells locate in the cell sheet capillaries (Scale bar $20 \mu \mathrm{m}$ ).

Arrow heads indicate the red blood cells. (g) The newly created vessel shape was cast by epoxy resin (Scale bar, $500 \mu \mathrm{m})$. (h) Expanded view of the resin cast of the newly created microvessels (Scale bar, $300 \mu \mathrm{m}$ ).

insights into strategies for producing clinically important tissue surrogates with new in vitro bioreactor designs, including: (1) in vitro engineered 3-D tissue surrogates with requisite biomechanical properties for eventually treating cardiac deficiencies, and (2) cardiac tissue-like models for applications in drug toxicity screening, biomechanics, transport physiology, improved tissue phantoms, and many other biomedical studies.

\section{Discussion}

The process of functional microvessel formation in the constructs is illustrated in Fig. 6a-e. The illustrations show that endothelial cells from the cell sheets migrated to the bioreactor microchannels and 
a

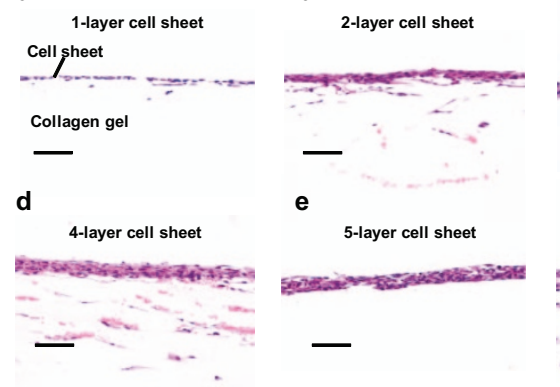
$9 \begin{gathered}\text { 6-layer cell sheet } \\ \text { (Double-steps) }\end{gathered}$

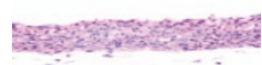

n
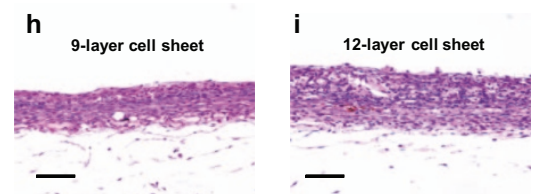$$
\text { j }
$$

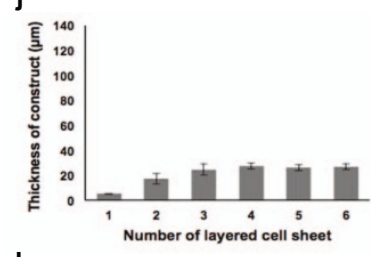

\section{I}
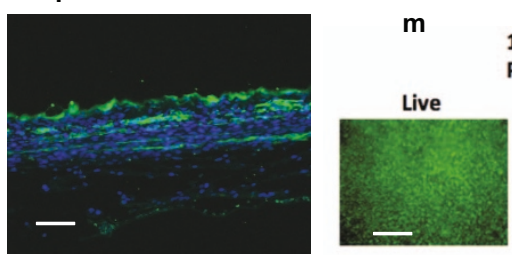

12 sheet Perfusion culture Dead
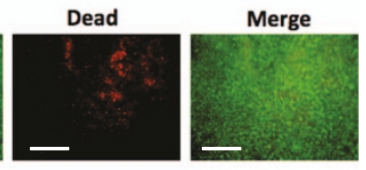

Figure 5 Fabrication of layered cardiac cell sheets on a perfusion bioreactor. In photographs $(\mathrm{a}) \sim(\mathrm{f})$, the upper layers are $1 \sim 6$-layered cell sheets, and the lower parts are collagen gel. (a) The thickness single-layered cell sheet shows $5 \pm 0.5 \mu \mathrm{m}$ in the bioreactor after 5 days cultivation;

(b) double-layered cell sheet is $17 \pm 4 \mu \mathrm{m}$; (c) triple-layered cell sheet is $24 \pm 4 \mu \mathrm{m}$; (d) quadruple-layered cell sheet is $27 \pm 2 \mu \mathrm{m}$;

(e) quintuple-layered cell sheet is $26 \pm 2 \mu \mathrm{m}$; (f) sextuple-layered cell sheet is $27 \pm 2 \mu \mathrm{m}$. (g) The thickness of the sextuple-layered cell sheet is $35 \pm 2 \mu \mathrm{m}$. (h) The thickness of quintuple-layered cell sheet is $65 \pm 8 \mu \mathrm{m}$. (i) Four triple-layered cell sheets layered up to four times at 5-day intervals produced a twelve layer sheet. The thickness of the twelve-layered cell sheet is $110 \pm 4 \mu \mathrm{m}$. (a) (i) (Scale bar, $50 \mu \mathrm{m}$ ). (j) Graph shows tissue thickness of the constructed grafts with a single-step layered procedure. The sheet thickness increased linearly up to triple-layered cell sheet and reached a plateau in quadruple to sextuple-layered cell sheets. (k) Graph shows that the tissue thickness of layered cell sheets increased up to a twelve-layered cell sheet with a multi-layered procedure. (1) Troponin T staining demonstrates the stratified cardiac muscle in a 6-layer cardiac cell sheet by the double-step procedure. Troponin T, Green; blue, nuclei (Scale bar, $20 \mu \mathrm{m})$. (m) Live/Dead assay was performed on the perfusion culture for the 12-layer cardiac cell sheet. Fluorescence micrographs show cells stained with calcein-AM (green-live; top images) and ethidium homodimer-1 (red = dead; middle images). Bottom images are merged from the Live/Dead images. A Live/Dead assay showed that most of the cells survived, but that focal cell death was observed (Scale bar, $20 \mu \mathrm{m}$ ).

consistently formed new vasculature-like structures in the collagen gel. This new vasculature eventually contributed substantially to cell transport, limited necrosis typically seen in thicker in vitro cultured cell masses, and provides new opportunities for improving ischemic limitations in regenerated tissue $\mathrm{e}^{15,16}$. Evidence supports the utility of the bioreactor system to induce vasculature formation by applying both fluidic shear stresses ${ }^{17,18}$ and cytokine gradients within the collagen gel ${ }^{19,20}$. Essential to overcoming the recognized limitations of constructing 3-D tissues in vitro, triple-layer cell sheets were repeatedly layered over the pre-integrated cell sheets within the perfused reactor. Each subsequent cell sheet layer spontaneously integrated with the existing construct and was rapidly infiltrated with budding vasculature extending from the previously vascularized cell sheets in the bioreactor. As each new cell sheet layer was added it was perfused with fresh medium flowing through the newly created vessels. This unique microvessel cultivation and rapid recruitment into newly integrated cell sheets avoids necrotic complications and reliably nourishes the thicker cell sheets that would be unable to survive in diffusion-limited transport culture conditions.

We sequentially performed the multi-step procedure up to four times and successfully fabricated 12-layer constructs, however Live/ Dead cell viability assays revealed focal cell death (Fig. $5 \mathrm{~m}$ ). When the procedures are repeated with the same cultivation conditions, the resulting vascular structure formation and media perfusion may be insufficient for whole tissue survival. Therefore, further biological and mechanical optimization (e.g. increasing flow rate in accordance with increasing tissue thickness) in the perfusion culture will be needed for making tissues thicker and more viable.

In summary, we have successfully fabricated thick, vascularized cardiac tissue-like surrogates with a new multi-step bioreactor and microchannel perfusion procedure in vitro. This approach spontaneously initiates then reliably builds an effective microvessel transport network between the growing cell sheets and the designed collagen-gel base. The in vitro bioreactor design improves on previously reported in vivo vascular bed formation for thick cell sheets ${ }^{3,5}$ by eliminating the tedious and impractical in vivo incubation steps. Importantly, the new method enables production of thick tissue constructs with pre-integrated and vessel-populated architectures that should provide new opportunities to contribute to more effective organ engineering, including highly perfused, high metabolic capacity tissues in the heart, liver, and kidney. This strategy is presented to contribute several new design and creation concepts, as well as practical production insight for future in vitro vascularized tissue surrogates, promising significant advances to both preclinical and clinical applications of tissue engineering.

\section{Methods}

All animal experiments were performed according to the "Guidelines of Tokyo Women's Medical University on Animal Use”. (The institutional approved ethics protocol \# 12-10).

Preparation of neonatal rat cardiac cell sheets. Neonatal rat cardiomyocytes were isolated from ventricles of 1-day-old rats (CLEA, Tokyo, Japan) using previously reported procedures ${ }^{21}$. The resulting cell suspensions were seeded at a density of $3.6 \times 10^{6}$ cells/dish on a temperature-responsive cell culture dish $\left(\mathrm{UpCell}^{\mathrm{TM}} \mathrm{dish}, \mathrm{I}\right.$.D $35 \mathrm{~mm}$, Type-E, CellSeed, Tokyo, Japan). Seeded dishes were incubated for 4 days. Confluent cells were harvested as a uniform single, intact and viable cell sheet by incubating the culture dishes in a $\mathrm{CO}_{2}$ incubator set at $20^{\circ} \mathrm{C}$ for 1 hour ${ }^{22,23}$.

Collagen gel support base with microchannels. Stainless steel wires (diameter $300 \mu \mathrm{m}$ ) were inserted into holes (I.D. $400 \mu \mathrm{m}$ ) in a culture device made by a rapid prototyping system (EDEN) (Objet Geometries, Billerica, MA, USA) (Fig. 1a). Neutralized collagen solution $(0.5 \%$, made from $10 \%$ decuple-concentrated cardiac cell culture medium described above), $10 \%$ balanced solution containing $10 \mathrm{mmol} / \mathrm{L}$ Hepes (Sigma, St. Louis, USA), $15 \mathrm{mmol} / \mathrm{L} \mathrm{NaHCO}_{3}$, and $0.5 \%$ collagen Type- 1 (IAC-05) (KOKEN, Tokyo, Japan) was poured into the culture device with aligned stainless wires held in parallel. To obtain a hard collagen gel, the collagen solution was incubated for $30 \mathrm{~min}$ at $37^{\circ} \mathrm{C}$. The steel wires were extracted from the mold after collagen gelation, yielding $300 \mu \mathrm{m}$-diameter channels in the collagen gel. Simultaneously with the collagen gelation, the first triple-layer cell sheet was placed on the collagen gel base containing the microchannels. The distance from the gel-formed microchannels to the overlaying cell sheets was approximately $500 \mu \mathrm{m}$.

Bioreactor assembly and perfusion culture condition. To stabilize the collagen-gel base, the collagen-gel base with the 3-layer cell sheet was cultured aseptically at $37^{\circ} \mathrm{C}$ for 1 hour. Next, the collagen-gel base was connected to a micro-syringe pump (KDS270) (KD Scientific, Holliston, USA). All bioreactor devices were placed in a culture box (As one, Tokyo, Japan) and maintained at $37^{\circ} \mathrm{C}$ by a fan heater (Sankei, 

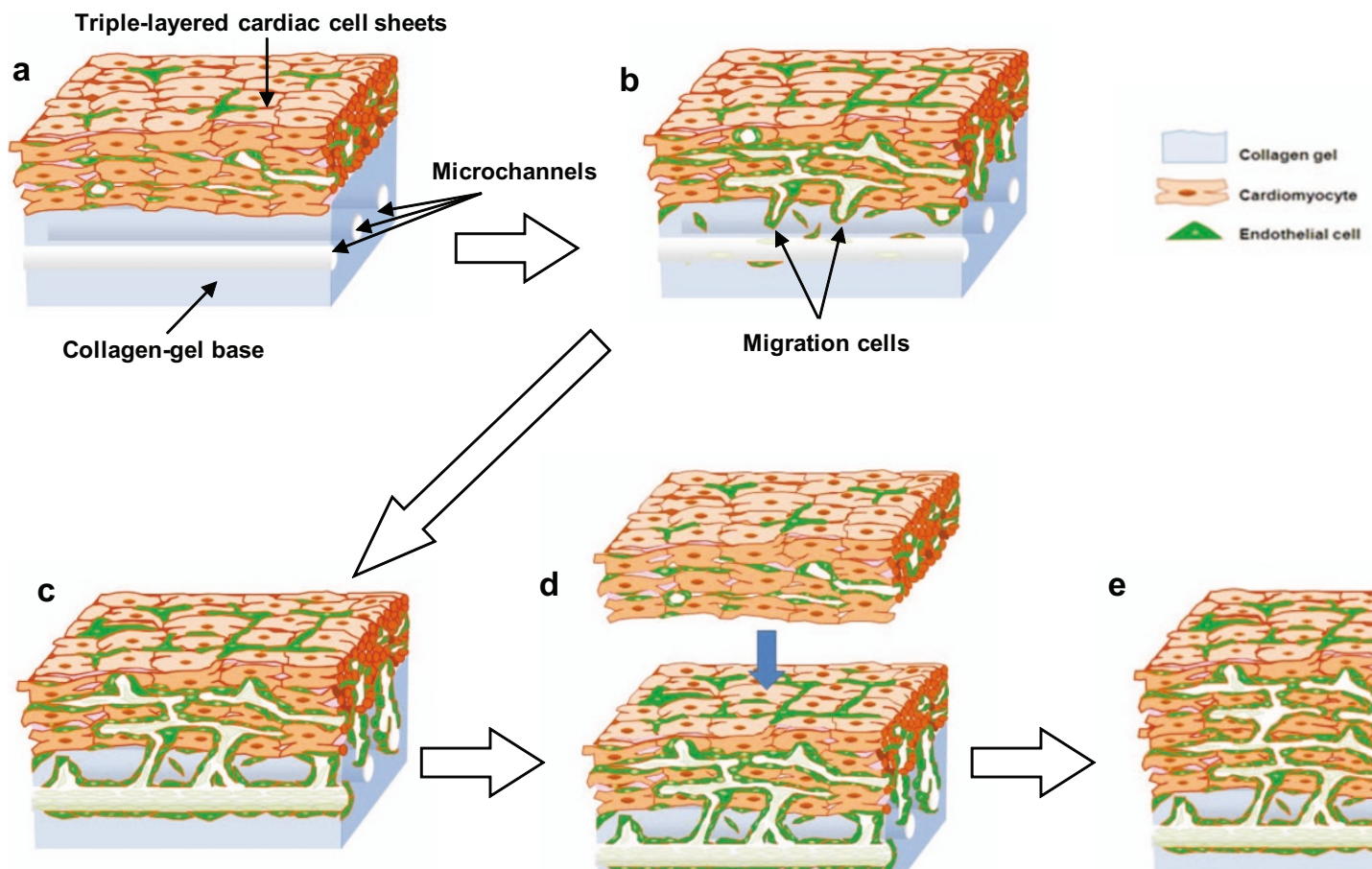

Vascularized tissue
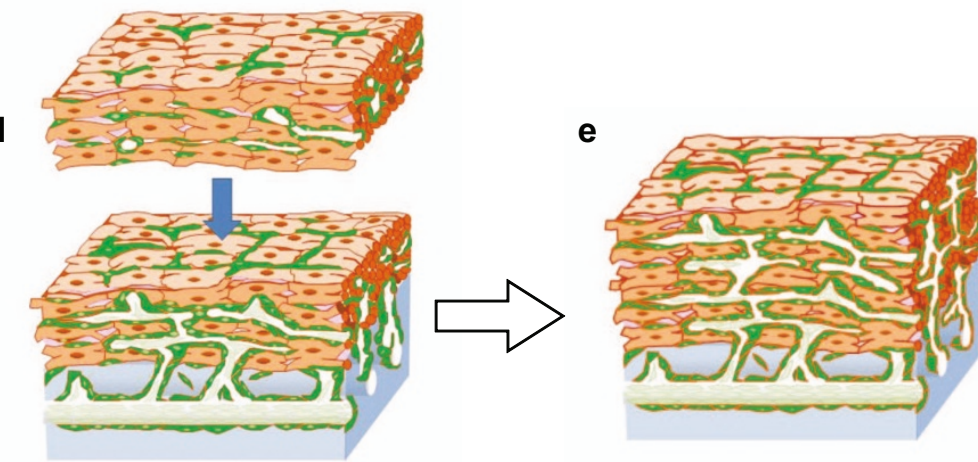

Another triple-layer

cardiac cell sheets

Vascularized thick 3D tissue

Figure 6 | Fabrication process of vascularized cardiac tissue in vitro. The illustrations show the process of vascularization of multi-layer cardiac cell sheets on collagen-based microchannels. (a) A triple-layered cardiac cell sheet on a collagen-gel base with microchannels. (b) After 5 days cultivation with perfusion through the microchannels, endothelial cells migrated into the collagen gel and formed a lumen structure. (c) New lumen-like vascular network was able to connect to the collagen-based microchannels. Fresh medium could flow into the new vascular network and to the vessels in the triple-layer cardiac cell sheets. (d) After new microvessels formed connecting with the collagen-gel microchannels, another triple-layered cardiac cell sheet was placed on the existing cell sheet. (e) The newly-layered cell sheet spontaneously integrated with the existing cell sheet and was also rapidly infiltrated with budding vasculature extending from the previously vascularized cell sheets in the bioreactor. By repeating the sheet layering process, the subsequently added cell sheets were perfused with fresh medium through the newly-created vessels.

Tokyo, Japan) then supplied with a mixed gas to maintain $\mathrm{pH} 7.4\left(5 \% \mathrm{CO}_{2}, 95 \%\right.$ air$)$. The perfusion rate of the culture medium was $0.5 \mathrm{~mL} / \mathrm{min}$ (Fig. 1b). The perfusion culture medium was comprised of $6 \%$ fetal bovine serum, $40 \%$ medium $199,1 \%$ penicillin-streptomycin solution, and $54 \%$ balanced salt solution: containing $166 \mathrm{mmol} / \mathrm{L} \mathrm{NaCl}, 1.0 \mathrm{mmol} / \mathrm{L} \mathrm{NaH}_{2} \mathrm{PO}_{4}, 0.8 \mathrm{mmol} / \mathrm{L} \mathrm{MgSO}_{4}, 26.2 \mathrm{mmol} / \mathrm{L}$ $\mathrm{NaHCO}_{3}, 0.9 \mathrm{mmol} / \mathrm{L} \mathrm{CaCl}, 5 \mathrm{mmol} / \mathrm{L}$ glucose, $50 \mathrm{ng} / \mathrm{mL}$ bFGF, and $50 \mathrm{ng} / \mathrm{mL}$ VEGF.

Histological analysis. After removal from the culture devices, multi-layer cell sheet constructs were fixed with $4 \%$ paraformaldehyde (Wako Pure Chemicals, Osaka, Japan) and routinely processed into $10-\mu \mathrm{m}$-tick paraffin-embedded sections. Hematoxylin and eosin, and Azan stained sections were prepared by conventional methods and examined by optical microscope.

To confirm perfusable blood vessels, a 1:10 diluted rat blood was perfused. For detecting GFP expressing endothelial cells, the constructs were frozen and sectioned. To detect cardiomyocytes, cross-sections were immunolabeled with a 1/100 dilution of anti-troponin T antibody (Thermo Scientific, CA, USA) for 2 hours at RT, and secondary stained for 2 hours at RT with Alexa Fluor 488-conjugated anti-mouse IgG anti- body for green fluorescence.

Analysis of migrating cells. The images of the AZAN-stained sections of the constructs were taken in situ by a microscope (ECLIPSE E800, Nikon, Tokyo, Japan). The area containing cells that migrated from the cell sheet was measured with ImageJ software. All data are expressed as mean \pm SD. An unpaired Student's t-test was performed to compare the two groups. One-way ANOVA was used for multiple group comparisons. If the F-distribution was significant, a Fisher's LSD test was used to specify differences between groups. A p-value of less than 0.05 was considered significant.

Live/Dead assay for process viability. A layered cell sheet was used to characterize cell viability after the fabrication process. After cultivation in the bioreactor, the constructs were submerged in a Live/Dead staining solution $(5 \mu \mathrm{mol} / \mathrm{L}$

calcein-AM-green and $0.5 \mu \mathrm{mol} / \mathrm{L}$ ethidium homodimer-1, Invitrogen, CA, USA) in a $\mathrm{pH}$-adjusted buffer. The submerged constructs were allowed to incubate for
30 minutes at $37^{\circ} \mathrm{C}$ and $5 \% \mathrm{CO}_{2}$. Fluorescence images were acquired using a microscope (OV-110, Olympus, Tokyo, Japan).

Separation of endothelial cells from primary isolated cardiac cells. To observe the behavior of endothelial cells in the myocardial cell sheets, the endothelial cells were first removed and replaced with GFP-expressing endothelial cells by magnetic cell sorting (MACS). For separating endothelial cells from normal rats or GFP-expressing rats (SLC, Hamamatsu, Japan), primary myocardial cell suspensions were incubated with mouse monoclonal anti-rat $\mathrm{CD} 31$ antibody. After rinsing with running buffer (PBS containing 5\% BSA and $2 \mathrm{mM}$ EDTA), cells were incubated with anti-mouse IgG-conjugated microbeads (Miltenyi Biotec GmbH, Bergisch Gladbach, Germany) and then washed again with running buffer. The labeled cells were applied to a LS column in the magnetic fields of a MiniMACS system (Miltenyi Biotec $\mathrm{GmbH}$, Bergisch Gladbach, Germany).The column was then washed with running buffer and removed from the magnetic fields, and the trapped cells in the column were flushed out with running buffer. Endothelial cell-depleted cardiomyocyte suspensions were used to create cell sheets without endothelial cells. To fabricate myocardial cell sheets containing GFP-positive endothelial cells, GFP-negative cardiac cells (without endothelial cells), and purified GFP-positive endothelial cells were mixed in a ratio of $8: 1$.

Resin perfusion for observation of lumen shape. To create a cast of the shape of the newly created vascular networks, an epoxy resin (MERCOX II, VT, USA) was perfused into the microchannels in the collagen-gel base. This resin is a modified acrylic casting material consisting of two components, a resin and a catalyst. After the liquid resin was mixed with a catalyst $(20: 1)$, it was perfused into the microchannels for 5 minutes, then the constructs were treated using $5 \% \mathrm{NaOH}$ to remove the collagen-gel and the cell sheets.

1. Levenberg, S. et al. Engineering vascularized skeletal muscle tissue. Nature Biotechnology 23, 879-884 (2005).

2. Melero-Martin, J. M. et al. Engineering robust and functional vascular networks in vivo with human adult and cord blood-derived progenitor cells. Circulation Research 103, 194-202 (2008). 
3. Morritt, A. N. et al. Cardiac tissue engineering in an in vivo vascularized chamber. Circulation 115, 353-360 (2007).

4. Shimizu, T. et al. Fabrication of pulsatile cardiac tissue grafts using a novel 3 -dimensional cell sheet manipulation technique and temperature-responsive cell culture surfaces. Circulation Research 90, e40-e48 (2002).

5. Shimizu, T. et al. Polysurgery of cell sheet grafts overcomes diffusion limits to produce thick, vascularized myocardial tissues. The FASEB Journal 20, 708 (2006).

6. Nishida, K. et al. Corneal reconstruction with tissue-engineered cell sheets composed of autologous oral mucosal epithelium. New England Journal of Medicine 351, 1187-1196 (2004).

7. Ohashi, K. et al. Engineering functional two-and three-dimensional liver systems in vivo using hepatic tissue sheets. Nature Medicine 13, 880-885 (2007).

8. Miyahara, Y. et al. Monolayered mesenchymal stem cells repair scarred myocardium after myocardial infarction. Nature Medicine 12, 459-465 (2006).

9. Ohki, T. et al. Treatment of oesophageal ulcerations using endoscopic transplantation of tissue-engineered autologous oral mucosal epithelial cell sheets in a canine model. Gut 55, 1704 (2006).

10. Haraguchi, Y., Shimizu, T., Yamato, M., Kikuchi, A. \& Okano, T. Electrical coupling of cardiomyocyte sheets occurs rapidly via functional gap junction formation. Biomaterials 27, 4765-4774 (2006).

11. Sasagawa, T. et al. Design of prevascularized three-dimensional cell-dense tissues using a cell sheet stacking manipulation technology. Biomaterials 31, 1646-1654.

12. Shimizu, T., Sekine, H., Yamato, M. \& Okano, T. Cell sheet-based myocardial tissue engineering: new hope for damaged heart rescue. Current Pharmaceutical Design 15, 2807-2814 (2009).

13. Carrier, R. L. et al. Cardiac tissue engineering: cell seeding, cultivation parameters, and tissue construct characterization. Biotechnology and Bioengineering 64, 580-589 (1999).

14. Carrier, R. L. et al. Effects of oxygen on engineered cardiac muscle. Biotechnology and Bioengineering 78, 617-625 (2002).

15. Koh, W., Stratman, A. N., Sacharidou, A. \& Davis, G. E. In vitro three dimensional collagen matrix models of endothelial lumen formation during vasculogenesis and angiogenesis. Methods in Enzymology 443, 83-101 (2008).

16. Arnaoutova, I., George, J., Kleinman, H. K. \& Benton, G. The endothelial cell tube formation assay on basement membrane turns 20: state of the science and the art. Angiogenesis 12, 267-274 (2009).

17. Hahn, C. \& Schwartz, M. A. Mechanotransduction in vascular physiology and atherogenesis. Nature Reviews Molecular Cell Biology 10, 53-62 (2009).

18. Wang, N. et al. Shear stress regulation of $\mathrm{Kr}$ ppel-like factor 2 expression is flow pattern-specific. Biochemical and Biophysical Research Communications 341 , 1244-1251 (2006).
19. Augustin, H. G., Koh, G. Y., Thurston, G. \& Alitalo, K. Control of vascular morphogenesis and homeostasis through the angiopoietin-Tie system. Nature Reviews Molecular Cell Biology 10, 165-177 (2009).

20. Adams, R. H. \& Alitalo, K. Molecular regulation of angiogenesis and lymphangiogenesis. Nature Reviews Molecular Cell Biology 8, 464-478 (2007).

21. Kinugawa, K. et al. Transcriptional regulation of inducible nitric oxide synthase in cultured neonatal rat cardiac myocytes. Circulation Research 81, 911 (1997).

22. Okano, T., Yamada, N., Sakai, H. \& Sakurai, Y. A novel recovery system for cultured cells using plasma-treated polystyrene dishes grafted with poly (N-isopropylacrylamide). Journal of Biomedical Materials Research 27, 1243 1251 (1993).

23. Yamato, M., Konno, C., Utsumi, M., Kikuchi, A. \& Okano, T. Thermally responsive polymer-grafted surfaces facilitate patterned cell seeding and co-culture. Biomaterials 23, 561-567 (2002)

\section{Acknowledgements}

This research is supported by a grant from the Japan Society for the Promotion of Science (JSPS) through the "Funding Program for World-Leading Innovative R\&D on Science and Technology (FIRST Program)," initiated by the Council for Science and Technology Policy (CSTP). We also appreciate the useful comments and technical criticism from Prof. W. Grainger (Colorado State University, USA) and Mr. Allan Nisbet.

\section{Author contributions}

K. Sakaguchi designed and conducted experiments, analyzed data and wrote the paper. T. Shimizu designed and conducted experiments, analyzed data and wrote the paper, and supervised this project. S. Horaguchi and $\mathrm{H}$. Sekine designed and conducted experiments M. Yamato, M. Umezu, and T. Okano supervised the project.

\section{Additional information}

Supplementary information accompanies this paper at http://www.nature.com/ scientificreports

Competing financial interests: T. Shimizu and M. Yamato are consultants for CellSeed, Inc. T. Okano is an investor in CellSeed, Inc., and is an inventor/developer designated on the patent for temperature-responsive culture surfaces.

License: This work is licensed under a Creative Commons Attribution 3.0 Unported License. To view a copy of this license, visit http://creativecommons.org/licenses/by/3.0/ How to cite this article: Sakaguchi, K. et al. In Vitro Engineering of Vascularized Tissue Surrogates. Sci. Rep. 3, 1316; DOI:10.1038/srep01316 (2013) 